\title{
El orden Carnivora (Mammalia) en el Perú: Estado del conocimiento y prioridades de investigación para su conservación
}

\author{
The order Carnivora (Mammalia) in Peru: State of Knowledge and research \\ priorities for its conservation
}

\begin{abstract}
E. Daniel Cossíos ${ }^{1,2}$, Paloma Alcázar ${ }^{3,4}$, Ursula Fajardo ${ }^{5}$, Kelly Chávez $^{6}$, Joanna Alfaro-Shigueto $^{7,8}$, Susana Cárdenas-Alayza ${ }^{9}$, Juan Valqui ${ }^{4,10}$, Francesca G. Montero ${ }^{11}$, Jesús Lescano ${ }^{12}$, Miryam Quevedo ${ }^{12}$, Elena Vivar ${ }^{5}$, Renata Leite ${ }^{13}$, Karim Ledesma ${ }^{4}$, César Medina ${ }^{6}$, Leonardo Maffei $^{14}$, Jessica Amanzo ${ }^{15}$, César Chávez ${ }^{16}$, Marco A. Enciso ${ }^{17}$, Álvaro García ${ }^{16}$, Jeffrey C. Mangel $^{7,8}$, Joel A. Mendoza ${ }^{18}$, Gianmarco Rojas ${ }^{18}$, Larissa Silva ${ }^{19}$, L. Villegas ${ }^{20}$, Robert S.R. Williams $^{19}$, Alfonzo Zúñiga ${ }^{21}$, Alex Cruz $^{22}$, IMARPE ${ }^{23}$, Elisa Ruiz ${ }^{24}$, DGFFS $^{24}$
\end{abstract}

$\begin{array}{ll}\text { Presentado: } & 20 / 10 / 2011 \\ \text { Aceptado: } & 28 / 04 / 2012\end{array}$ Publicado online: $01 / 10 / 2012$
1: University of Geneva. 30 Quai Ernest Ansermet 1211, Genève 4, Switzerland.

2: Kawsay Pacha-Asociación Biodiversidad. Av Caminos del Inca 2436, Surco, Lima, Perú.

3: Universidad Ricardo Palma, Escuela Académico Profesional de Ciencias Veterinarias, Facultad de Ciencias Biológicas. Av. Benavides 5440, Surco. Lima, Perú.

4: Centro de Ornitología y Biodiversidad (CORBIDI). Calle Santa Rita 105 Of. 202, Surco. Lima 33, Perú.

5: Museo de Historia Natural de la Universidad de San Marcos, Av. Arenales 1256, Jesús María. Lima, Perú.

6: Museo de Historia Natural de la Universidad Nacional San Agustín. Av. Alcides Carrión s/n. Arequipa, Perú.

7: Pro Delphinus. Enrique Palacios 630-204, Lima 11, Perú.

8: Centre for Ecology and Conservation, School of Biosciences, University of Exeter, Cornwall Campus, Penryn, UK.

9: Centro para la Sostenibilidad Ambiental, Universidad Peruana Cayetano Heredia. Av. Armendariz \# 445, Miraflores. Lima, Perú.

10: Instituto de Zoología, Universidad de Kiel. Am Botanischen Garten 1-9, 24118 Kiel, Alemania.

11: Universidad Nacional Agraria La Molina. Av. La Molina s/n, La Molina, Lima.

12: Laboratorio de Anatomía Animal y Fauna Silvestre, Facultad de Medicina Veterinaria, Universidad Nacional Mayor de San Marcos. Av. Circunvalación Cdra. $28 \mathrm{~s} / \mathrm{n}$ - San Borja. Lima, Perú.

13: Center for Tropical Conservation, Duke University. 3705 Erwin Rd, Durham, NC. USA.

14: Wildlife Conservation Society. Arias Araguez 152, Miraflores. Lima, Perú.

15: Laboratorio de Estudios en Biodiversidad. Departamento de Ciencias Biologicas y Fisiologicas, Facultad de Ciencias y Filosofia, Universidad Peruana Cayetano Heredia. Honorio Delgado 430, Urb. Ingenieria, SMP, Lima, Perú.

16: Centro Neotropical de Entrenamiento en Humedales Internacional. Calle David Lewellyn 870, Coquimbo, Región de Coquimbo, Chile.

17: Departamento de Reprodução Animal. Faculdade de Medicina Veterinária e Zootecnia. Universidade de São Paulo. Av. Prof. Dr. Orlando Marques de Paiva n87. Cidade Universitária. São Paulo/SP. Brasil.

18: Parque Zoológico Huachipa. Av Las torres s/n Ate Vitarte. Lima, Perú.

19: Frankfurt Zoological Society. Clorinda Matto de Turner 305, Urb. Magisterio, Cusco.

20: Instituto Regional de Ciencias Ambientales, Universidad Nacional de San Agustín, Arequipa.

21: Word Wildlife Fund. Perú.

22: Alianza Gato Andino. Av. Ejército 1349, Tacna. Perú.

23: Instituto del Mar del Perú. Esquina Gamarra y General Valle s/n, Chucuito. Callao, Perú.

24: Dirección General Forestal y de Fauna Silvestre del Ministerio de Agricultura. Calle Diecisiete 355, urb El Palomar, San Isidro. Lima, Perú.

\section{Resumen}

La alta diversidad de especies de carnívoros del Perú puede generar problemas al momento de decidir los taxa y temas sobre los que deben dirigirse los esfuerzos de investigación. En este trabajo se evalúa el esfuerzo de investigación en base al número de publicaciones realizadas para cada familia y especie de carnívoro en el Perú. Asimismo, se señalan los vacíos de información relevantes para la conservación de cada especie y se presenta la primera evaluación de las prioridades de investigación sobre este grupo animal en el Perú. Se registró 145 publicaciones sobre carnívoros peruanos realizadas desde el año 1943. El número de publicaciones presentó grandes diferencias entre taxa, entre temas estudiados y entre las ecorregiones en las que se realizaron las investigaciones. Según la escala de prioridades propuesta, la especie que debe ser estudiada con mayor prioridad es el coatí andino Nasua olivacea y la de menor prioridad es el ocelote Leopardus pardalis. Los resultados de nuestro trabajo resaltan la urgencia de realizar investigaciones sobre ciertas especies de carnívoros de las que existen pocos datos publicados, tanto a nivel local como global, y que se distribuyen en pocas ecorregiones del Perú. Tanto la escala de prioridades de investigación como la lista de vacíos de información serán de utilidad para guiar esfuerzos logísticos y financieros de investigadores particulares, instituciones privadas y gubernamentales.

Palabras clave: estado de conocimiento, carnívoros, diversidad, conservación, Perú.

\section{Abstract}

The high diversity of Peruvian carnivore species may pose problems when deciding to which taxa and topics new research efforts should be directed. In this publication, we evaluated the research effort made on each taxa -by assessing the number of publications per species-, point out the knowledge gaps that are important to the conservation of each species and present the first evaluation of research priorities for this group of animals to the country. We registered 145 publications about Peruvian carnivores made since 1943. The number of publications is significantly different between taxa, between subjects and between ecoregions where the research was conducted. According to the proposed priority scale, the species to be studied with greater priority is Nasua olivacea and the lowest priority is for Leopardus pardalis. The results of our study highlight the urgent need to conduct research on certain species of carnivores about which there are few published data, locally and globally, and which occupy a low number of ecoregions in the country. Both the scale of research priorities and the list of knowledge gaps presented here will be useful to guide logistical and financial efforts, for individual researchers as well as for private or governmental institutions. 


\section{Introducción}

El orden Carnivora es uno de los grupos de animales que ha llamado más fuertemente la atención del ser humano (Ginsberg 2001) y, con 71 especies amenazadas a nivel global (UICN 2011), constituye uno de los taxa en mayor peligro del mundo. Debido a sus grandes necesidades de espacio y de calidad de hábitat, los carnívoros son frecuentemente considerados "especies sombrilla” es decir aquellas especies cuya conservación debería asegurar la de muchos otros organismos, y son también "especies carismáticas", por ser capaces de atraer fácilmente la simpatía de las personas. Estas características hacen que los carnívoros cumplan un rol importante como objetivo central de programas de conservación de vida silvestre. Sin embargo, para que un programa de conservación sea efectivo, requiere de información sobre la distribución, el estado poblacional, los requerimientos ecológicos y la identidad genética de las especies implicadas (Wilson 2000).

Pacheco et al. (2009) reportaron 34 especies de carnívoros que ocurren en el Perú, de las cuales una, el lobo fino de Juan Fernández (Arctocephalus philippii), sólo lo hace ocasionalmente (Majluf \& Reyes 1989). A éstas hay que agregar el elefante marino del sur (Mirounga leonina), también de ocurrencia ocasional en el país (Campagna 2008), con lo que el número de especies del orden Carnivora en el Perú llega a 35. Esto equivale al 12,4\% de las 281 especies vivientes del orden Carnivora a nivel mundial (Wilson \& Mittermeier 2009), lo que resalta la importancia de la conservación de este taxón en el Perú.

Al momento de decidir qué investigaciones realizar para la elaboración de programas de conservación o para, simplemente, aumentar nuestro conocimiento sobre el orden Carnivora, la gran diversidad de especies de este taxón en el Perú plantea un problema: ¿por dónde empezar? Al existir limitaciones de tiempo, recursos financieros y humanos, es importante tener una idea de qué proyectos de investigación podrían ser más útiles o urgentes. Como varios autores han expresado, las investigaciones y otros esfuerzos con fines de conservación deberían dirigirse con prioridad a las especies más amenazadas (e.g. Carter et al. 2000, Dunn 2002) pero, de forma evidente, es necesario tomar también en cuenta el grado de conocimiento actual sobre cada especie, siendo prioritario estudiar los taxa sobre los cuales existe menos información disponible.

En este trabajo se señalan los vacíos de información relevantes para la conservación de los carnívoros existentes en el Perú y se presenta la primera evaluación de las prioridades de investigación sobre este grupo animal para el país. Esta evaluación fue desarrollada como una herramienta para la toma de decisiones de la Dirección General Forestal y de Fauna Silvestre (DGFFS) del Ministerio de Agricultura peruano. Puesto que puede realizarse tanto investigaciones dirigidas a una sola especie como a varias especies que se encuentren en una misma área, se realizaron dos evaluaciones de prioridades de investigación: una dirigida a especies individuales de carnívoros y otra dirigida a ecorregiones. Con el fin de que dichas evaluaciones sean replicables con otros grupos taxonómicos y de que se pueda hacer incluso comparaciones entre especies de diferentes grupos, se empleó un método cuantitativo para su realización, dándose prioridad a las especies más amenazadas y a aquellas de las que se tiene menos información.

\section{Material y métodos}

Se realizó una búsqueda de literatura sobre cada especie de carnívoro en los índices de Zoological Record (Thomson Reuters), las bases de datos de internet ISI Web of Knowledge (apps.webofknowledge.com) y Google Scholar (scholar.google. com), los índices de revistas peruanas orientadas a la biología (i.e. Revista Peruana de Biología, Ecología Aplicada, The Biologist) y en bibliotecas de distintas universidades e institutos peruanos. La búsqueda incluyó artículos en revistas científicas, libros, tesis y literatura gris (i.e. informes de instituciones gubernamentales y privadas). Este material bibliográfico fué revisado para determinar qué temas fueron estudiados y cuáles son los vacíos de información trascendentes para la conservación en el caso de 33 de las 35 especies de carnívoros presentes en el Perú. El lobo fino de Juan Fernández y el elefante marino del sur, ambos de ocurrencia ocasional en el país, fueron las dos únicas especies de carnívoros que no fueron incluidas en la revisión bibliográfica ni en los análisis posteriores.

Para cada publicación, se registró las especies de carnívoros estudiadas, las ecorregiones en las que se realizó la investigación y el tema estudiado. La nomenclatura empleada fue la de Pacheco et al. (2009). Las ecorregiones fueron registradas siguiendo la clasificación de Brack-Egg (1986). Los temas estudiados fueron separados en las seis categorías siguientes: i) distribución, ii) ecología y comportamiento, iii) amenazas y relaciones con el ser humano, iv) salud, v) genética de poblaciones y taxonomía subespecífica y vi) tamańo, tendencia y densidad poblacional. Se confeccionó una lista de las publicaciones sobre carnívoros peruanos sin tomar en cuenta: i) las publicaciones duplicadas (e.g. traducciones de artículos o tesis originales), ii) publicaciones monográficas, resultantes de la reunión de datos publicados con anterioridad, iii) publicaciones sobre temas distintos a los expuestos más arriba (e.g. arquelogía, paleontología, biología celular o molecular), iv) datos anecdóticos (i.e. observaciones casuales y reportes hechos sin base científica), v) listas de diversidad biológica. Adicionalmente, se revisaron los resúmenes de los congresos de la Sociedad Peruana de Mastozoología, del Instituto de Investigación de Ciencias Biológicas Antonio Raimondi (ICBAR) y de los congresos de Conservación y Educación ambiental realizados en el Perú entre los ańos 1999 y 2010. Los datos de los resúmenes de congresos sirvieron para determinar qué temas han sido estudiados pero no publicados aún, es decir que los resúmenes no fueron considerados publicaciones. Se determinó los grupos taxonómicos y temas que han sido más estudiados y se analizó la evolución del esfuerzo en investigación como el número de publicaciones sobre carnívoros realizadas por ańo.

La determinación de las prioridades de investigación se abordó de dos formas. En primer lugar, se elaboró una lista de vacíos de información sobre cada especie, separando éstos en i) vacíos principales y ii) otros vacíos de información, considerándose como principales a los vacíos de información que deberían ser llenados de forma urgente para la elaboración de planes de conservación. Para esta separación se tomó en cuenta que la urgencia de los datos sobre un mismo tema puede ser diferente en cada especie, dependiendo de la información disponible, del estado de conservación y de distintas características de cada especie. Así, por ejemplo, será más urgente determinar el impacto de la caza sobre un carnívoro tradicionalmente perseguido por el ser humano que para otro que no soporta esa amenaza de forma 
habitual. El listado de vacíos de información y su separación en "principales" y "otros" fueron realizados, en la medida de lo posible, por gente con experiencia de investigación en cada especie analizada.

La segunda forma de determinar las prioridades de investigación fue elaborar una escala de prioridad para especies y otra de prioridad de investigación para ecorregiones distintas. La escala para especies siguió un método modificado a partir de Lucherini et al. (2004). Cada especie recibió un puntaje para cuatro variables y se sumaron estos cuatro puntajes para obtener el valor de prioridad de investigación de la especie. El puntaje dado a cada variable tuvo un valor máximo de 1 para lograr que todas las variables tuvieran el mismo peso en el puntaje final. Las variables elegidas y la forma de puntuarlas fueron las siguientes:

a.i) Selectividad de hábitat: Dado que los carnívoros peruanos se encuentran distribuidos sobre nueve ecorregiones ( $\mathrm{Pa}$ checo et al 2009), un puntaje entre $1 / 9$ y 1 fue asignado a cada especie, según el número de ecorregiones en la que se le encuentre. El objetivo de este puntaje fue dar prioridad a las especies de distribución más restringida. A una especie presente en las nueve ecorregiones le correspondió así el puntaje de $1 / 9$, mientras que a otra presente en una sola ecorregión se le dio un puntaje de 1 .

a.ii) Vulnerabilidad: Durante 2010 y 2011, la DGFFS organizó una serie de talleres para la recategorización de la fauna amenazada del Perú, siguiendo la metodología de la Unión Internacional para la Conservación de la Naturaleza (UICN). La categoría de amenaza propuesta para cada especie determinó el puntaje de vulnerabilidad, otorgándose el mayor valor a las especies más amenazadas. Se dio un punto a las especies categorizadas como en peligro crítico (CR), $5 / 6$ a aquellas en peligro (EN), $2 / 3$ a las vulnerables (VU), $1 / 2$ a las de datos insuficientes (DD), $1 / 3$ a las casi amenazadas (NT) y $1 / 6$ a las de bajo riesgo (LR).

a.iii) Esfuerzo de investigación hecho a nivel nacional: El propósito de este punto fue que las especies para las que se ha estudiado menos aspectos a nivel nacional reciban un mayor puntaje. Se consideró los seis temas o tipos de investigación registrados y se dio $1 / 6$ de punto por cada tema sobre el cual no existe información publicada. Así, las especies para las que no existen datos publicados sobre ninguno de esos aspectos obtuvieron un punto, mientras que aquellas para las que los seis aspectos han sido estudiados obtuvieron un puntaje de 0 .

a.iv) Esfuerzo de investigación a nivel global: De forma parecida al punto anterior, se dio un puntaje del 0 al 1 dependiendo de los aspectos que han sido estudiados a nivel global.

Para crear la escala de prioridad de investigación por ecorregiones, se escogieron tres variables y se sumó el puntaje de todas para cada ecorregión, con un método modificado también a partir de Lucherini et al (2004). Las variables elegidas y la forma de puntuarlas fueron las siguientes:

b.i) Vulnerabilidad promedio de las especies de carnívoros presentes en una ecorregión: De 1/6 a 1, siguiendo los valores dados en el punto a.ii.

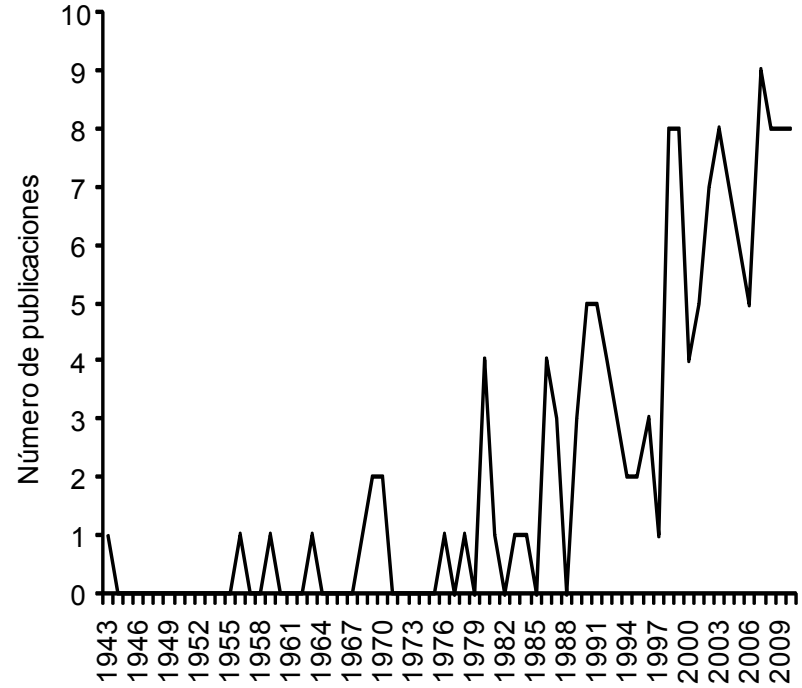

Figura 1. Evolución del número de publicaciones sobre carnívoros en el Perú desde el año 1943.

b.ii) Diversidad de carnívoros: Se dio un punto por cada especie de carnívoro presente en una ecorregión. Dado que el número máximo de especies de carnívoros en una ecorregión es de 18 (en la selva baja; Pacheco et al. 2009), y con la intención de que el puntaje máximo sea igual a 1 , se dividió el total obtenido entre 18 .

b.iii) Representatividad de la investigación realizada: Para cada ecorregión se calculó el número $t$, igual al total de temas susceptibles a ser estudiados, multiplicando el número de especies de carnívoros presentes en la ecorregión por seis (el número de tipos de investigación posibles). Se otorgó entonces un puntaje de $1 /$ t por cada tema que no ha sido estudiado, obteniéndose valores posibles entre $0 \mathrm{y}$ 1, mayores mientras menos investigación sobre carnívoros se haya hecho en una ecorregión.

Para probar la robustez del método empleado (i.e. la capacidad del método de quedar inafectado frente a factores de distorsión), se cuantificaron los cambios que ocurren en el orden de la escala de prioridad al doblar el peso de cada una de las variables escogidas.

\section{Resultados}

\section{A. Publicaciones sobre el orden Carnivora en el Perú.}

Desde 1943 hasta 2010 han sido publicados 145 trabajos en 80 artículos de revistas, 16 libros o capítulos de libro, 29 tesis universitarias y 20 informes además de, al menos, 46 resúmenes de congresos y eventos académicos. La publicación más antigua registrada correspondió al año 1943. Desde entonces, el número de publicaciones sobre carnívoros peruanos se ha ido incrementando gradualmente (Fig. 1).

La familia de carnívoros con mayor número de publicaciones fue Otariidae, con 59 (40,7\% del total de publicaciones), mientras que Procyonidae, con una sola publicación, fue la que tuvo menos. Luego de los otáridos, la familia con más publicaciones fue Mustelidae, con 32 (22,1\%; Fig. 2), aunque 29 de ellas estuvieron dedicadas a sólo 2 de sus 8 especies: la nutria marina Lontra felina y el lobo de río, Pteronura brasiliensis.

A nivel de especies, el mayor número de publicaciones 


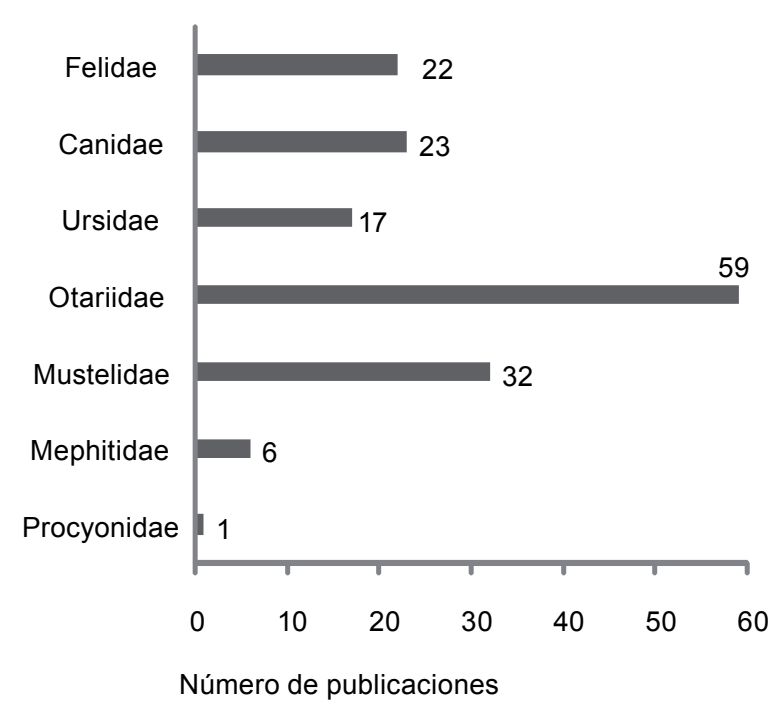

Figura 2. Número de publicaciones por familia del orden Carnivora, realizadas entre 1943 y 2010.

estuvo dirigida al lobo marino fino Arctocephalus australis, con 51 (35,2\% de las publicaciones), mientras que no se encontró ninguna publicación para 13 especies de carnívoros. Sin tener en cuenta a los otáridos, la especie con más publicaciones fue el oso de anteojos Tremarctos ornatus (17 publicaciones), seguido por la nutria marina (15), el lobo de río (14) y el zorro andino Lycalopex culpaeus (13) (Fig. 3).

Entre los temas tratados, el que tuvo mayor número de publicaciones fue el de ecología y comportamiento, con 75 (51,7\% del total de publicaciones) y el tema con un menor número fue el de salud, con 8 (5,5\%; Fig. 4). La mayor parte de las investigaciones fue realizada en la ecorregión de Desierto Costero (77 publicaciones o $53,1 \%$ ), mientras que las ecorregiones menos estudiadas fueron el Bosque Pluvial del Pacífico y la Sabana de Palmeras, con cero y una publicación, respectivamente (Fig. 5).

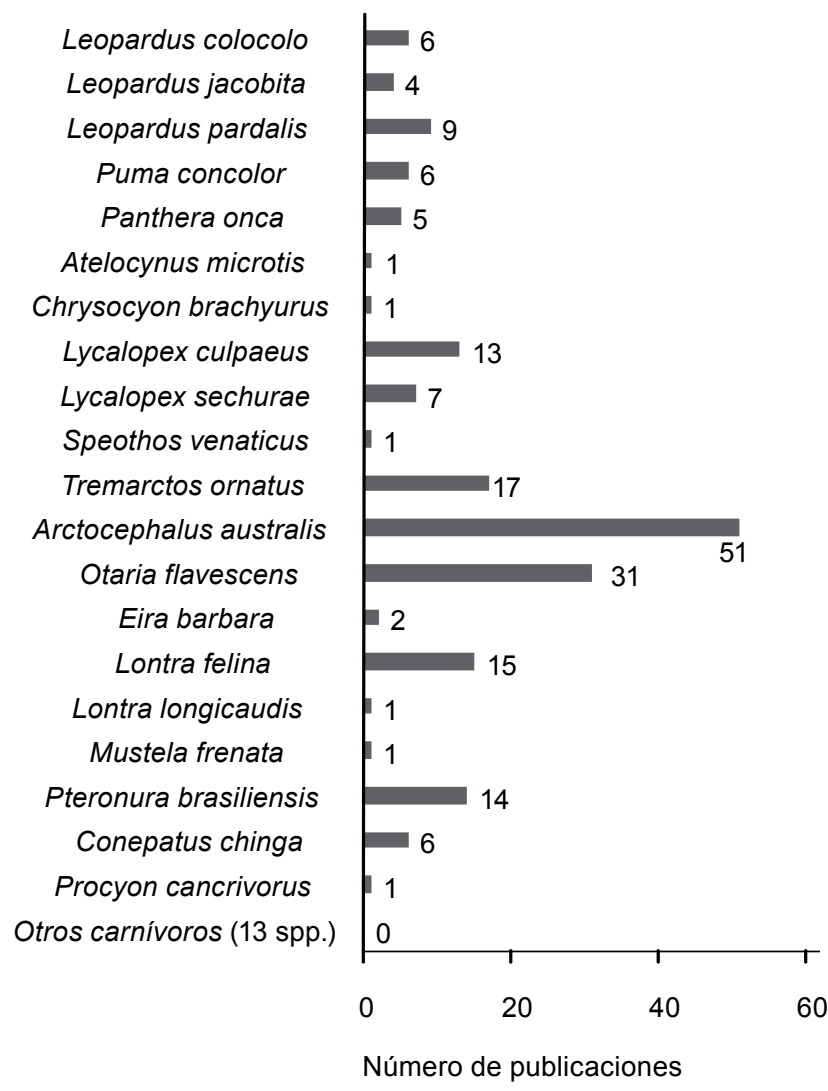

Figura 3. Número de publicaciones por especie del orden Carnivora, realizadas entre 1943 y 2010.

Sin tener en cuenta a los otáridos, la ecorregión más estudiada fue la Selva Baja, con 28 publicaciones (31,1\%; Fig. 5).

De los 46 resúmenes de congresos encontrados, 27 (58,7\%) presentaron información que no ha sido publicada hasta el momento en artículos, libros o tesis y seis de ellos tocaron, en

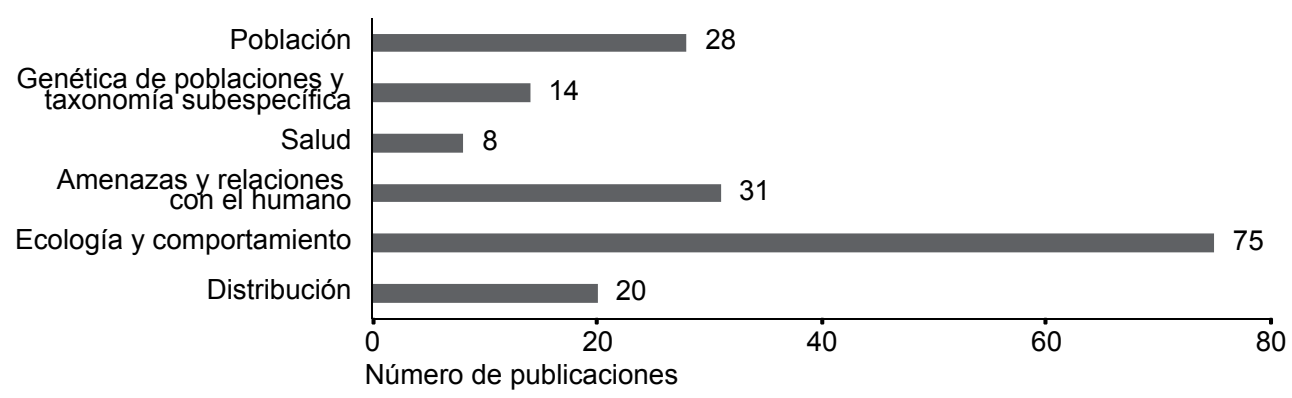

Figura 4. Número de publicaciones sobre carnívoros peruanos realizadas entre 1943 y 2010, clasificadas por tema de investigación tratado.

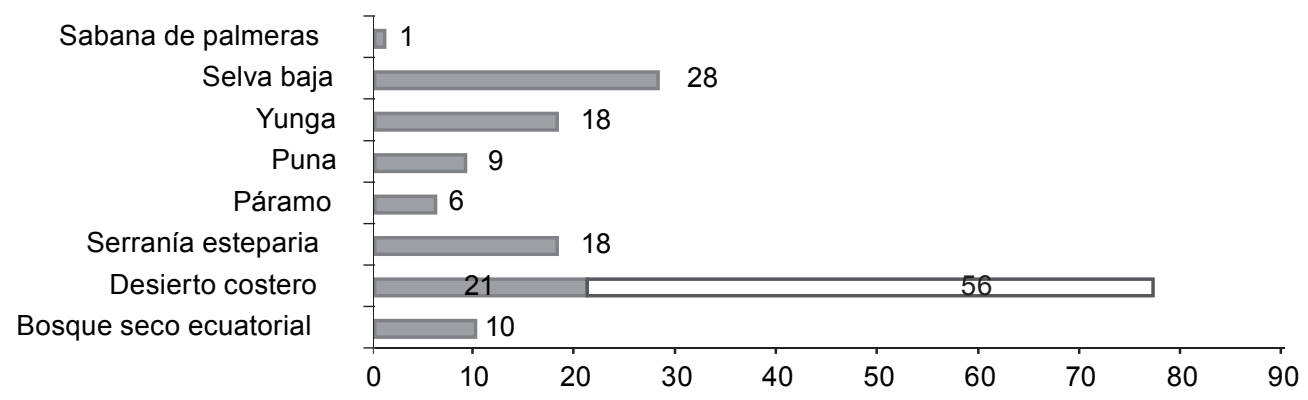

Figura 5. Número de publicaciones sobre carnívoros peruanos realizadas entre 1943 y 2010, clasificadas por ecorregión en la que se realizó la investigación. En Desierto costero: blanco representa las publicaciones sobre otáridos, gris claro representa las publicaciones sobre otros carnívoros. 
relación a alguna especie de carnívoro en particular, temas o ecorregiones sobre los que no existe información publicada aún.

\section{B. Temas estudiados y vacíos de información}

Los vacíos de información identificados, divididos en "principales" y "otros" para cada especie, fueron los siguientes:

\section{Gato de pajonal, Leopardus colocolo}

Temas estudiados: Distribución, relaciones con el ser humano y genética poblacional en gran parte del país (entre La Libertad y Tacna). Un sólo estudio de dieta, basado en 9 muestras fecales.

Vacíos principales: Distribución en el norte del país, flujo genético entre poblaciones, datos poblacionales en general. Otros vacíos: Ecología en general (incluyendo dieta, horario de actividad, uso de hábitat y relaciones con otros carnívoros), datos sobre reproducción y salud.

\section{Gato andino, Leopardus jacobita}

Temas estudiados: Distribución, genética de poblaciones y relaciones con el ser humano entre Ancash y Tacna.

Vacíos principales: Se desconoce el límite norte de su distribución y su presencia en distintas zonas de Ancash, Pasco, Huancavelica y Ayacucho debe ser confirmada. Flujo genético entre poblaciones. Abundancia, densidad poblacional y área de actividad. Otros vacíos: ecología en general, reproducción y salud.

\section{Ocelote, Leopardus pardalis}

Temas estudiados: Comportamiento, dieta, densidad poblacional y amenazas en selva baja. Un sólo registro de parásitos.

Principales vacíos: Distribución, datos poblacionales y cuantificación del impacto de amenazas, principalmente de actividades antrópicas. Otros vacíos: Estado sanitario y posibles impactos de la introducción de patógenos de origen doméstico.

\section{Oncilla, Leopardus tigrinus}

Principales vacíos: Distribución, amenazas, datos poblacionales. Otros vacíos: Ecología en general, epidemiología y genética poblacional.

\section{Margay, Leopardus wiedii}

Principales vacíos: Distibución, datos poblacionales y ecología en general. Estimación del impacto de amenazas de origen antrópico, incluyendo la modificación y pérdida de hábitat, la cacería de subsistencia y el uso de esta especie con fines curativos, mágico religiosos y folklóricos. Otros vacíos: No se han realizado estudios sobre las subespecies $L$. w. pirrensis y $L . w$. amazonica, ambas presentes en el Perú según Cabrera (1958), y determinado si realmente son poblaciones reproductivamente aisladas. Estado sanitario.

\section{Puma, Puma concolor}

Temas estudiados: Dieta en selva baja, bosque seco y páramo (de ese último ambiente, sólo 9 muestras fecales). Uso de hábitat en bosque seco. Relaciones con el ser humano en Ayacucho y en el Parque Nacional Cordillera Azul. Un sólo registro de parásitos.

Principales vacíos: Cuantificación de conflictos con el ser humano y del valor económico y ecológico de este carnívoro. Es- tudios sobre medidas de control de conflictos. Hace falta estimar abundancia y densidad poblacional en distintos ambientes y más estudios sobre su ecología. Otros vacíos: Presión de caza sobre el puma y sus principales presas. Estudios de distribución. Identificación de corredores entre poblaciones. Evaluación del uso de esta especie con fines curativos, mágico-religiosos o folclóricos. Evaluación sanitaria que incluya agentes infecciosos presentes en el puma y agentes en animales domésticos y humanos. El MINAM (2011) considera importante determinar, mediante métodos genéticos, si existen poblaciones aisladas y pequeñas, cuya conservación sea prioritaria.

\section{Yaguarundi, Puma yagouaroundi}

Principales vacíos: Distibución, datos poblacionales y ecología en general. Se requiere identificar las amenazas que soporta esta especie y estimar su impacto, así como identificar las áreas de mayor importancia para su conservación (i.e. aquellas en las que se encuentre poblaciones mayores y saludables). Otros vacíos: División a nivel subespecífico (principalmente por métodos genéticos), datos reproductivos, estado sanitario.

\section{Jaguar, Panthera onca}

Temas estudiados: Dieta y densidad de posibles presas en selva baja. Se ha incluído muestras de jaguares peruanos de Loreto, únicamente, en un estudio de genética poblacional a nivel sudamericano.

Principales vacíos: Es necesario completar la información sobre la distribución de la especie, abundancia poblacional, ecología básica y disponibilidad de presas. Se necesita documentar la ocurrencia de conflictos con seres humanos y ganadería para comprender su extensión e importancia a nivel local. Otros vacíos: Estudios sanitarios tanto en cautiverio como en vida libre. El Ministerio del Ambiente del Perú-MINAM (2011) consideran importante determinar, mediante métodos genéticos, si existen poblaciones aisladas y pequeńas.

\section{Perro de monte de orejas cortas, Atelocynus microtis}

Temas estudiados: Observaciones preliminares (de estudios aun no publicados) sobre dieta, comportamiento y densidad poblacional en el Parque Nacional de Manu.

Principales vacíos: El conocimiento acerca de su distribución en el país aún se encuentra incompleto. No se tiene estimación alguna de densidad, tendencia o tamaño poblacional, se desconocen aspectos básicos de su ecología y como consecuencia, se entiende poco acerca de sus requerimientos de hábitat. Otros vacíos: La biología y patología de esta especie son poco o nada conocidas.

\section{Lobo de crin, Chrysocyon brachyurus}

Temas estudiados: Los únicos datos publicados sobre dieta (un sólo estómago examinado), distribución y actividad horaria corresponden al primer reporte de la especie para el Perú.

Principales vacíos: Datos poblacionales y relación de la población peruana con poblaciones aledańas. Otros vacíos: Dado que casi no se ha hecho investigación sobre esta especie en el Perú, cualquier proyecto sobre ecología o conservación sería valioso. 


\section{Zorro andino, Lycalopex culpaeus}

Temas estudiados: Distribución. Dieta en serranía esteparia, lomas costeras y yunga. Uso de hábitat, parasitología y relaciones con el ser humano en serranía esteparia. Comportamiento horario en yunga.

Principales vacíos: Se desconoce los límites de distribución de las dos subespecies que, presuntamente, existen en el Perú (L. c. smithersi y L. c. andina) y tampoco se les ha diferenciado genéticamente. Se requiere datos sobre dinámica poblacional en las principales zonas donde ocurren conflictos con el ser humano, para poder establecer medidas de control, monitoreo y conservación. Es necesaria una descripción de las relaciones con el ser humano, una cuantificación de los daños hacia los animales domésticos a lo largo del país y un estudio de la efectividad de posibles medidas de control. No existen publicaciones de estudios en cautiverio ni sobre reproducción. Fuera de la dieta, se desconocen los demás aspectos de su ecología y biología. Otros vacíos: Relación con otros carnívoros, principalmente con el zorro de Sechura (Lycalopex sechurae) y zorro gris (Lycalopex griseus). Se tiene muy pocos datos sobre enfermedades y su rol en la epidemiología de zoonosis, como la hidatidosis.

\section{Zorro gris, Lycalopex griseus}

Principales vacíos: Distribución, tamaño poblacional y relación de la población peruana con las de otros países. Conflictos con el ser humano. Se desconoce el límite norte y el límite altitudinal en el país. Existe la posibilidad de que la población peruana deba catalogarse como una especie nueva o como una nueva subespecie de L. griseus, (com pers. Elena Vivar). Otros vacíos: Relación con otros predadores, sobre todo con $L$. culpaeus, con el que está en simpatría en muchas zonas de la costa sur. No existen estudios sobre la ecología de esta especie en el Perú, ni estudios en cautiverio ni datos sobre enfermedades que la afecten. Se desconoce el rol que pueda tener este cánido en la epidemiología de la hidatidosis humana y otras zoonosis.

\section{Zorro de Sechura, Lycalopex sechurae}

Temas estudiados: Distribución. Relaciones con el ser humano. Dieta, dispersión de semillas y actividad horaria en bosque seco.

Principales vacíos: Datos poblacionales, genética de poblaciones y taxonomía subespecífica. No se conoce enfermedades que afecten a esta especie pero se sospecha que éstas pueden ser causantes de grandes pérdidas poblacionales, además de ser un riesgo potencial para la salud humana. Otros vacíos: Se desconoce el límite sur (Grimwood 1969, Pacheco 2002) y el límite altitudinal de su distribución en el Perú. Reproducción, sus relaciones con otros predadores; sobre comportamiento sólo se ha realizado observaciones en un único individuo juvenil en cautiverio (Birdseye 1956).

\section{Perro de monte, Speothos venaticus}

Temas estudiados: Observaciones preliminares sobre dieta y comportamiento.

Principales vacíos: Es necesario estimar su distribución y densidad poblacional, así como determinar las amenazas que soporta (MINAM 2011). Se sugiere especialmente determinar qué tan susceptible es este cánido a los cambios de hábitat y a la presión de caza sobre sus presas, así como estudiar el impacto de enfermedades transmitidas por animales domésticos. Otros vacíos: Dado que se ha hecho muy pocas observaciones sobre esta especie en el Perú, cualquier proyecto sobre ecología o conservación sería valioso.

\section{Oso de anteojos, Tremarctos ornatus}

Temas estudiados: Distribución, amenazas, relaciones con el ser humano y ecología en general. Datos poblacionales en yunga.

Principales vacíos: Distribución en los departamentos de Amazonas, Ayacucho, Huancavelica y Puno. Estudios poblacionales, tanto a nivel nacional como regional. Dada la heterogeneidad de la disponibilidad de recursos en los Andes, es necesario determinar áreas representativas que permitan un menor error en la extrapolación de los valores de abundancia. Informacion sobre variación genética a nivel nacional. Identificación de las poblaciones más amenazadas debido a fragmentacion de hábitat y aislamento. Impacto de agentes patógenos de animales domésticos. Mortalidad natural, por caceria y por enfermedades introducidas.

\section{Lobo marino fino, Arctocephalus australis}

Temas estudiados: Distribución, amenazas y relaciones con el ser humano, población, ecología en general, salud, genética de poblaciones a gran escala.

Principales vacíos: Magnitud e impacto de las capturas ilegales e incidentales, área de actividad (home range) en más de una localidad, dispersión entre colonias reproductivas, actualizar datos de dieta durante ańos Niño y no-Niño, dinámica y estructura poblacional durante y después de eventos El Niño, ecotoxicología, estructura genética de las poblaciones peruana y chilena, epidemiología, zoonosis, parasitología, estudios sanitarios, estudios locales de disponibilidad de presa. Otros vacíos: Características de los viajes de alimentación (profundidad de buceo, distancias recorridas), comportamiento y dispersión de juveniles.

\section{Lobo marino chusco, Otaria flavescens}

Temas estudiados: Distribución, amenazas y relaciones con el ser humano, población, ecología en general, salud.

Principales vacíos: Magnitud e impacto de las capturas ilegales e incidentales, impacto de las pesquerías industriales, área de actividad en más de una localidad, dispersión entre colonias reproductivas, interacción entre lobos chuscos y pesca en diferentes localidades, tasa de supervivencia de crías, adultos y juveniles, actualizar datos dieta durante años Niño y no-Niño, dinámica y estructura poblacional, ecotoxicología, epidemiología y estudios sanitarios en general, estudios locales de disponibilidad de presas. Otros vacíos: Características de los viajes de alimentación (profundidad de buceo, distancias recorridas), comportamiento y dispersión de juveniles.

\section{Manco, Eira barbara}

Temas estudiados: Una sola publicación, con estimaciones poblacionales para selva baja.

Principales vacíos: Datos poblacionales, identificación de amenazas y cuantificación de sus impactos (MINAM 2011). Otros vacíos: Biología y ecología. Estudios epidemiológicos y parasitológicos. 


\section{Hurón menor, Galictis cuja y}

\section{Hurón grande, Galictis vittata}

Principales vacíos: Distribución, datos poblacionales, amenazas. Otros vacíos: Ecología en general, comportamiento, enfermedades y genética poblacional.

\section{Gato marino, Lontra felina}

Temas estudiados: Distribución, población, amenazas y relaciones con el ser humano, ecología en general, comportamiento, genética de poblaciones.

Principales vacíos: Se requiere una estimación que actualice los datos poblacionales del año 2004, área de actividad, estudios sobre enfermedades y parasitología, identificación de zonas que podrían estar contaminadas por patógenos, impacto del fenómeno "El Niño". Otros vacíos: Se requiere profundizar los estudios de genética poblacional para identificar posibles unidades de manejo.

\section{Nutria, Lontra longicaudis}

Temas estudiados: Una sola publicación, con estimaciones poblacionales para selva baja.

Principales vacíos: Datos poblacionales. Efectos de amenazas, principalmente de la caza, enfermedades transmitidas por animales domésticos y modificación del hábitat. Otros vacíos: Biología y ecología, enfermedades en general, genética poblacional.

\section{Comadreja amazónica, Mustela africana}

Principales vacíos: Distribución, requerimientos ambientales y amenazas. Otros vacíos: Biología y ecología en general, genética poblacional.

\section{Comadreja andina, Mustela frenata}

Temas estudiados: Relaciones con el ser humano en Ayacucho.

Principales vacíos: Identificación de amenazas y cuantificación de sus impactos. Otros vacíos: Biología, ecología y enfermedades en general, datos poblacionales, genética poblacional.

\section{Lobo de río, Pteronura brasiliensis}

Temas estudiados: Distribución, población, amenazas y relaciones con el ser humano, ecología en general.

Principales vacíos: Identificación de las poblaciones reproductivas en los departamentos de Loreto y Ucayali, evaluación de conflictos con pescadores de subsistencia y pescadores comerciales. identificación y monitoreo de impactos negativos y positivos del turismo, estimación del éxito de supervivencia de crías.

\section{Zorrino andino, Conepatus chinga $y$}

\section{Zorrino hocico de cerdo, Conepatus semistriatus}

Temas estudiados: En el caso de $C$. chinga, se ha estudiado la distribución y amenazas en el sur del Perú, y dieta en un bosque de Arequipa. Dos parásitos han sido registrados para el género Conepatus pero la especie de zorrino es dudosa.

Principales vacíos: Distribución, especialmente donde pueda haber solapamiento entre ambas especies, genética poblacional, principalmente para identificar poblaciones que requieran trata- miento especial. Otros vacíos: Se requiere saber si existe hibridación entre ambas especies. Dado que se ha hecho muy poca investigación sobre esta especie en el Perú, cualquier proyecto sobre ecología, datos poblacionales, amenazas y relaciones con el ser humano sería valioso.

\section{Olingo, Bassaricyon alleni}

Principales vacíos: Distribución, datos poblacionales y amenazas. Otros vacíos: Biología, ecología y enfermedades en general. Es de interés determinar si Bassaricyon alleni debe ser considerada una especie distinta a $B$. gabbi y a otras especies descritas de ese género. Si así fuera, el papel del Perú en la conservación de $B$. alleni cobraría mayor importancia, puesto que allí se encuentra la mayor parte de su área de distribución.

\section{Coatí de nariz blanca, Nasua narica}

Principales vacíos: Es necesario determinar su distribución, estado de su población y las amenazas que enfrenta en el Perú (MINAM 2011), así como la relación entre la población peruana y la que se encuentra en Ecuador. Entre las posibles amenazas sobre esta especie, se requiere determinar el posible impacto de la pérdida de hábitat, las relaciones que tendría con el ser humano y la transmisión de enfermedades a través de animales domésticos. Otros vacíos: Biología, ecología y enfermedades en general.

\section{Coatí de cola anillada, Nasua nasua}

Aunque se trata de una especie común, es necesario determinar la tendencia de sus poblaciones, identificar las amenazas que enfrenta y las áreas que mantienen poblaciones mayores. Otros Vacios: Información sobre biología, ecología y enfermedades en general. Estructura genética poblacional.

\section{Coatí andino, Nasuella olivacea}

Principales vacíos: Distribución, datos poblacionales y amenazas. Dado que la población registrada en Perú se encuentra aislada y muy distante del resto (en Venezuela, Colombia y Ecuador) es importante determinar, por métodos morfológicos y moleculares, si se le debe tratar como una especie distinta. Otros vacíos: Biología, ecología y enfermedades en general.

\section{Chosna, Potos flavus}

Principales vacíos: Como es una especie mascota, las enfermedades que pueden ser transmitidas al hombre deben ser estudiadas con mayor detalle. Otros vacíos: Ecología, biología, amenazas y genética poblacional.

\section{Osito cangrejero, Procyon cancrivorus}

Temas estudiados: Dos especies de parásitos han sido reportadas para este carnívoro en el Perú.

Principales vacíos: Distribución y amenazas. Otros vacíos: Biología, ecología y enfermedades en general. No se conoce la estructura genética de las poblaciones de esta especie; un estudio a nivel global sobre este tema permitiría saber, entre otras cosas, qué tan grande es la diferencia entre las poblaciones de la costa peruana y de otros países del Pacifico y las de la selva baja.

\section{Propuesta de escala de prioridades de investigación}

En la escala de prioridades de investigación por especie, los valores obtenidos variaron entre 3,33 y 1,06 puntos sobre 4,0 
posibles. La especie con mayor prioridad de investigación fue el coatí andino, mientras que la especie con menor prioridad fue el ocelote (Tabla 1). Al variar el peso del puntaje de las distintas variables, entre 8 y 11 especies cambiaron de lugar dentro de la escala de priorización, y se movieron entre 0,51 (al doblar el peso de la variable «esfuerzo de investigación a nivel nacional») y 1,58 puestos (al doblar el peso de la variable «vulnerabilidad») en promedio.

En la escala de prioridades de investigación por ecorregiones, los valores obtenidos variaron entre 2,98 y 2,23 puntos sobre 3,0 posibles. La ecorregión con mayor prioridad de investigación fue la selva baja, mientras que la ecorregión con menor prioridad fue el páramo (Tabla 2). Al variar el peso del puntaje de las distintas variables, entre 3 y 5 ecorregiones cambiaron de lugar dentro de la escala de priorización, y se movieron entre 0,67 (al doblar el peso de la variable "diversidad") y 0,89 puestos (al doblar el peso de las variables "vulnerabilidad" o "representatividad de la investigación”) en promedio.

\section{Discusión y conclusiones}

Existen grandes diferencias en el número de publicaciones hechas sobre las distintas especies de carnívoros peruanos. Los otáridos han sido los carnívoros más estudiados en el Perú, quizá debido a su permanencia en colonias durante parte del año -lo cual facilita su observación y captura- y a razones logísticas como una relativa facilidad para el desplazamiento de los investigadores hacia los lugares de observación y la existencia de una estación biológica en San Juan de Marcona, en el departamento de Ica, donde se ha hecho una parte importante de las investigaciones.

Entre los fisípedos, parece haber una preferencia por las especies amenazadas: las especies más estudiadas -oso de anteojos, lobo de río y nutria marina- son tres de los únicos cinco carnívoros peruanos considerados dentro de alguna categoría de amenaza a nivel global (IUCN 2011). Las otras dos especies amenazadas a ese nivel, el gato andino y la oncilla, con cuatro y ninguna publicación respectivamente, son félidos elusivos y quizá hayan sido dejados de lado debido a una mayor dificultad para conseguir datos sobre ellos. El siguiente grupo de carnívoros más estudiados está formado por siete especies con cuatro o más publicaciones cada una y que pueden ser consideradas relativamente comunes. Finalmente, se encuentra un grupo de 20 especies con una o ninguna publicación, todas ellas, con excepción de la oncilla, especies no incluidas en ninguna categoría de amenaza y, en su mayoría, de distribución restringida, aunque también hay algunas que pueden considerarse de distribución amplia. Un patrón similar se encuentra en las publicaciones sobre carnívoros de Brasil, que dan preferencia a las especies consideradas "bandera" y a aquéllas para las cuales es más fácil conseguir información (de Oliveira 2006).

Algunos autores han señalado la existencia de preferencias hacia especies carismáticas en desmedro de las especies amenazadas al momento de realizar investigaciones (e.g. Brodie 2009, Tisdell y Swarda Nantha 2007). En el caso de los carnívoros peruanos es posible que este factor también influya en las preferencias de los investigadores aunque, al no existir estimaciones del "carisma” de las especies implicadas, no es posible comprobarlo aún.

El número de trabajos presentados a congresos y reuniones científicas y cuyos resultados no han sido publicados hasta ahora, 27, es alto. Su publicación haría crecer la literatura sobre carní- voros peruanos en un $18,6 \%$ y, por lo tanto, mejoraría nuestro conocimiento sobre el tema. Muchas veces, la información presentada en resúmenes de congresos no basta para conocer o entender la metodología empleada ni para hacer replicable la experiencia expuesta, y no ha pasado por el filtro de calidad que supone su publicación en una revista científica; por esas razones, son muchas las revistas que no aceptan como referencia un resumen de congreso o un póster.

Los resultados de nuestro trabajo resaltan la urgencia de realizar investigación sobre ciertas especies de carnívoros y sobre ecosistemas específicos. Cabe resaltar que el sistema de clasificación de ecorregiones de Brack utilizado en este estudio considera al mar, la costa y al desierto costero dentro de una misma ecorregión (ecorregión del desierto costero). Al centrarse en los otáridos, que se distribuyen exclusivamente en el ámbito marino-costero, la mayoría de investigaciones sobre carnívoros peruanos se ha realizado en sólo una parte de la ecorregión del desierto costero. Según la escala de prioridades propuesta, los carnívoros que más urge investigar son especies sobre las que existen muy pocos datos publicados, tanto a nivel nacional como global, y que ocupan un bajo número de ecorregiones en el país. Varias de estas especies se encuentran en la categoría de datos insuficientes a nivel nacional y podrían estar realmente amenazadas. En el caso de las ecorregiones, la selva baja y la yunga se encuentran en los primeros puestos, influenciadas en gran medida por el alto número de especies que habitan en ellas.

Como todo sistema de priorización de investigaciones (e.g. Lucherini et al. 2004, de Oliveira 2006, Regan et al. 2008, Carter et al. 2000), el presentado aquí contiene un componente de subjetividad causado por la elección de variables y la importancia dada a cada una de ellas. El orden de prioridad de las especies o ecorregiones puede variar según el peso que se le dé a las variables escogidas. Sin embargo, el sistema es bastante robusto, presentando pocos cambios al elevarse el peso de cualquier variable al doble. En el caso de que un usuario decida dar preferencia absoluta a alguna de las variables (por ejemplo a la categoría de amenaza) bastaría con separar a las especies en categorías según la variable en cuestión y ver luego la prioridad de las especies dentro de cada categoría. Aún en el caso de elegirse, para hacer investigación, a un taxón determinado tomando en cuenta razones distintas a su estado de conservación y al nivel de conocimiento sobre él (por ejemplo el carisma de una especie o la facilidad para la toma de datos), la lista de vacíos de información presentada aquí debería ser de utilidad para decidir qué temas estudiar.

Es necesario resaltar que aún para las especies de carnívoros que han sido más estudiadas en el Perú se carece de información importante para su manejo y conservación, tanto en cuanto a temas como a áreas geográficas. En muchos casos, además, no basta con información aislada en el tiempo. Por ejemplo, en el caso de los carnívoros más estudiados, los otáridos, los cambios ambientales a los que están sujetos ocasionan fluctuaciones poblacionales importantes (Soto 1999, Soto et al. 2004), por lo que se requiere contar con información actualizada y de largo plazo. La sugerencia de prioridades hecha en esta publicación no pretende, por lo tanto, frenar el esfuerzo actualmente realizado hacia algunas especies y desviarlo hacia otras, pero puede servir de guía para nuevos proyectos de investigación. 
Tabla 1. Escala propuesta de prioridades de investigación en especies de carnívoros peruanos. Se muestra el puntaje dado a cada variable elegida para la evaluación, así como el puntaje total. Las especies se encuentran ordenadas según su prioridad, de mayor a menor.

\begin{tabular}{|c|c|c|c|c|c|c|c|c|c|c|c|}
\hline \multirow[b]{2}{*}{ 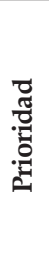 } & \multirow[b]{2}{*}{ Especie } & \multirow[b]{2}{*}{ Familia } & \multicolumn{2}{|c|}{$\begin{array}{c}\text { Selectividad } \\
\text { de hábitat }\end{array}$} & \multicolumn{2}{|c|}{ Vulnerabilidad } & \multicolumn{2}{|c|}{$\begin{array}{c}\text { Investigación a } \\
\text { nivel nacional }\end{array}$} & \multicolumn{2}{|c|}{$\begin{array}{l}\text { Investigación } \\
\text { a nivel global } \\
\end{array}$} & \multirow[b]{2}{*}{ 嵌 } \\
\hline & & & 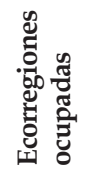 & 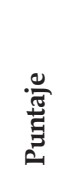 & 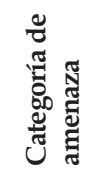 & $\begin{array}{l}\frac{.}{\pi} \\
\frac{\pi}{5} \\
\end{array}$ & 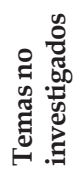 & $\begin{array}{l}\frac{0}{\pi} \\
\frac{\pi}{3} \\
\end{array}$ & 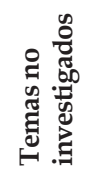 & 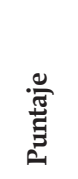 & \\
\hline 1 & Nasua olivacea & Procyonidae & 1 & 1,00 & DD & 0,50 & 6 & 1,00 & 5 & 0,83 & 3,33 \\
\hline 2 & Mustela africana & Mustelidae & 1 & 1,00 & LC & 0,17 & 6 & 1,00 & 6 & 1,00 & 3,17 \\
\hline 3 & Galictis cuja & Mustelidae & 1 & 1,00 & DD & 0,50 & 6 & 1,00 & 4 & 0,67 & 3,17 \\
\hline 4 & Atelocynus microtis & Canidae & 2 & 0,89 & VU & 0,67 & 5 & 0,83 & 4 & 0,67 & 3,06 \\
\hline 5 & Bassaricyon alleni & Procyonidae & 2 & 0,89 & $\mathrm{LC}$ & 0,17 & 6 & 1,00 & 6 & 1,00 & 3,06 \\
\hline 6 & Galictis vittata & Mustelidae & 1 & 1,00 & $\mathrm{LC}$ & 0,17 & 6 & 1,00 & 5 & 0,83 & 3,00 \\
\hline 7 & Leopardus wiedii & Felidae & 2 & 0,89 & DD & 0,50 & 6 & 1,00 & 3 & 0,50 & 2,89 \\
\hline 8 & Speothos venaticus & Canidae & 2 & 0,89 & DD & 0,50 & 5 & 0,83 & 4 & 0,67 & 2,89 \\
\hline 9 & Leopardus tigrinus & Felidae & 1 & 1,00 & DD & 0,50 & 6 & 1,00 & 2 & 0,33 & 2,83 \\
\hline 10 & Nasua narica & Procyonidae & 1 & 1,00 & LC & 0,17 & 6 & 1,00 & 4 & 0,67 & 2,83 \\
\hline 11 & Potos flavus & Procyonidae & 4 & 0,67 & DD & 0,50 & 6 & 1,00 & 4 & 0,67 & 2,83 \\
\hline 12 & Lontra longicaudis & Mustelidae & 3 & 0,78 & DD & 0,50 & 5 & 0,83 & 4 & 0,67 & 2,78 \\
\hline 13 & Puma yagouaroundi & Felidae & 3 & 0,78 & DD & 0,50 & 6 & 1,00 & 3 & 0,50 & 2,78 \\
\hline 14 & Procyon cancrivorus & Procyonidae & 3 & 0,78 & DD & 0,50 & 5 & 0,83 & 4 & 0,67 & 2,78 \\
\hline 15 & Nasua nasua & Procyonidae & 3 & 0,78 & $\mathrm{LC}$ & 0,17 & 6 & 1,00 & 4 & 0,67 & 2,61 \\
\hline 16 & Lycalopex griseus & Canidae & 2 & 0,89 & DD & 0,50 & 6 & 1,00 & 1 & 0,17 & 2,56 \\
\hline 17 & Leopardus jacobita & Felidae & 1 & 1,00 & EN & 0,83 & 3 & 0,50 & 1 & 0,17 & 2,50 \\
\hline 18 & Pteronura brasiliensis & Mustelidae & 2 & 0,89 & EN & 0,83 & 2 & 0,33 & 2 & 0,33 & 2,39 \\
\hline 19 & Conepatus semistriatus & Mephitidae & 6 & 0,44 & $\mathrm{LC}$ & 0,17 & 6 & 1,00 & 4 & 0,67 & 2,28 \\
\hline 20 & Eira barbara & Mustelidae & 5 & 0,56 & $\mathrm{LC}$ & 0,17 & 5 & 0,83 & 4 & 0,67 & 2,22 \\
\hline 21 & Lontra felina & Mustelidae & 1 & 1,00 & EN & 0,83 & 1 & 0,17 & 1 & 0,17 & 2,17 \\
\hline 22 & Lycalopex sechurae & Canidae & 3 & 0,78 & NT & 0,33 & 3 & 0,50 & 3 & 0,50 & 2,11 \\
\hline 23 & Mustela frenata & Mustelidae & 5 & 0,56 & $\mathrm{LC}$ & 0,17 & 5 & 0,83 & 3 & 0,50 & 2,06 \\
\hline 24 & Otaria flavescens & Otariidae & 1 & 1,00 & VU & 0,67 & 1 & 0,17 & 0 & 0,00 & 1,84 \\
\hline 25 & Chrysocyon brachyurus & Canidae & 1 & 1,00 & NT & 0,33 & 3 & 0,50 & 0 & 0,00 & 1,83 \\
\hline 26 & Arctocephalus australis & Otariidae & 1 & 1,00 & EN & 0,83 & 0 & 0,00 & 0 & 0,00 & 1,83 \\
\hline 27 & Panthera onca & Felidae & 5 & 0,56 & NT & 0,33 & 4 & 0,67 & 0 & 0,00 & 1,56 \\
\hline 28 & Conepatus chinga & Mephitidae & 4 & 0,67 & $\mathrm{LC}$ & 0,17 & 2 & 0,33 & 2 & 0,33 & 1,50 \\
\hline 29 & Lycalopex culpaeus & Canidae & 3 & 0,78 & $\mathrm{LC}$ & 0,17 & 2 & 0,33 & 1 & 0,17 & 1,44 \\
\hline 30 & Tremarctos ornatus & Ursidae & 5 & 0,56 & VU & 0,67 & 1 & 0,17 & 0 & 0,00 & 1,39 \\
\hline 31 & Leopardus colocolo & Felidae & 5 & 0,56 & DD & 0,50 & 2 & 0,33 & 0 & 0,00 & 1,39 \\
\hline 32 & Puma concolor & Felidae & 8 & 0,22 & DD & 0,50 & 3 & 0,50 & 0 & 0,00 & 1,22 \\
\hline 33 & Leopardus pardalis & Felidae & 5 & 0,56 & LC & 0,17 & 2 & 0,33 & 0 & 0,00 & 1,06 \\
\hline
\end{tabular}

Tabla 2. Escala propuesta de prioridades de investigación sobre carnívoros, por ecorregiones. Se muestra el puntaje dado a cada variable elegida para la evaluación, así como el puntaje total. Las ecorregiones se encuentran ordenadas según su prioridad, de mayor a menor.

\begin{tabular}{|c|c|c|c|c|c|c|c|c|c|c|}
\hline \multirow[b]{2}{*}{ 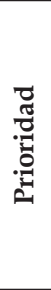 } & \multirow[b]{2}{*}{ Ecorregión } & \multicolumn{2}{|c|}{ Vulnerabilidad } & \multicolumn{2}{|c|}{ Diversidad } & \multicolumn{4}{|c|}{ Representatividad de investigación } & \multirow[b]{2}{*}{ 岕 } \\
\hline & & 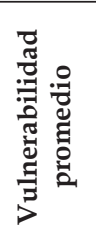 & 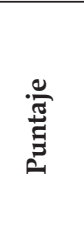 & 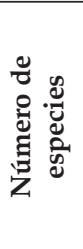 & 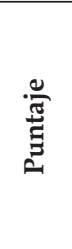 & + & 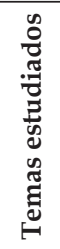 & 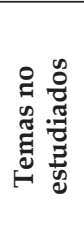 & $\begin{array}{l}\frac{0}{\pi} \\
\frac{\pi}{\Xi} \\
\Xi\end{array}$ & \\
\hline 1 & Selva baja & 0,37 & 1,12 & 18 & 1,00 & 108 & 15 & 93 & 0,86 & 2,98 \\
\hline 2 & Yunga & 0,36 & 1,09 & 16 & 0,89 & 96 & 10 & 86 & 0,90 & 2,88 \\
\hline 3 & Sabana de palmeras & 0,44 & 1,33 & 8 & 0,44 & 48 & 2 & 46 & 0,96 & 2,74 \\
\hline 4 & Bosque seco ecuatorial & 0,38 & 1,15 & 10 & 0,56 & 60 & 6 & 54 & 0,90 & 2,61 \\
\hline 5 & Desierto costero & 0,5 & 1,52 & 9 & 0,50 & 54 & 23 & 31 & 0,57 & 2,59 \\
\hline 6 & Bosque pluvial del Pacífico & 0,36 & 1,09 & 8 & 0,44 & 48 & 0 & 48 & 1,00 & 2,54 \\
\hline 7 & Puna & 0,41 & 1,24 & 9 & 0,50 & 54 & 13 & 41 & 0,76 & 2,50 \\
\hline 8 & Serranía esteparia & 0,35 & 1,06 & 11 & 0,61 & 66 & 12 & 54 & 0,82 & 2,49 \\
\hline 9 & Páramo & 0,38 & 1,15 & 5 & 0,28 & 30 & 6 & 24 & 0,80 & 2,23 \\
\hline
\end{tabular}




\section{Agradecimientos}

Agradecemos a V. Pacheco y al Ministerio del Ambiente por los comentarios hechos durante nuestra investigación. Un agradecimiento especial a M. Cossíos y a B. Veillon por su revisión del texto en sus versiones iniciales. El proceso de reunión y análisis de información fue apoyado técnica y financieramente por la DGFFS del Ministerio de Agricultura del Perú.

\section{Literatura citada}

Arias-Schreiber M \& C. Rivas. 1998. Distribución, tamaño y Estructura de las poblaciones de lobos marinos Arctocephalus australis y Otaria byronia en el Litoral Peruano, En Noviembre 1996 y Marzo 1997. Inf. Progr. Inst. Mar Perú, 73: 17-32.

Birdseye C. 1956. Observations on a domesticated Peruvian desert fox, Dusicyon. Journal of Mammalogy 37:284-287.

Brack-Egg E. 1986. Las ecorregiones del Perú. Boletín de Lima, 44:57-70.

Cabrera A. 1958. Catálogo de los mamíferos de América del Sur. I. (Metatheria, Unguiculata, Carnivora). Revista Museo Argentino de Ciencias Naturales Bernardino Rivadavia, Ciencias Zoológicas, 4:1-307.

Campagna C. 2008. Mirounga leonina. In: IUCN 2010. IUCN Red List of Threatened Species. Version 2010.4. Acceso 24/5/2011.

Carter M.F., W.C. Hunter, D.N. Pashley \& K.V. Rosenbery. 2000. Setting priorities for landbird conservation in the United States: the partners in flight approach. Auk 117:541-548.

Dunn E.H. 2002. Using decline in bird populations to identify needs for conservation action. Conservation Biology 16:1632-1637.

Ginsberg J.R. 2001. Setting priorities for carnivore conservation: What makes carnivores different? Pp: 498-523. In: Carnivore Conservation (Gittleman J. L., S.M. Funk, D.W. Macdonald, and R.K. Wayne, eds.). Cambridge University Press, Cambridge, 675 pp.

Grimwood R. 1969. Notes on the distribution and status of some Peruvian mammals 1968. Special Publication 21, New York Zoological Society, New York.
IUCN. 2011. IUCN Red List of Threatened Species. Version 2011.1. http://www.iucnredlist.org Acceso 27/7/2011.

Lucherini M., L. Soler, \& E. Luengos. 2004. A preliminary revision of knowledge status of felids in Argentina. Mastozoología Neotropical 11(1):7-17.

Majluf P. \& J.C. Reyes. 1989. The marine mammals of Peru: a review. In: Pauly, D, Muck, P, Mendo, J and Tsukayama, I. (eds.) The Peruvian Upwelling Ecosystem: Dynamics and Interactions. IMARPE-GTZ-ICLARM: 334-363

MINAM. 2011. Especies CITES de carnívoros peruanos. Ministerio del Ambiente, Dirección. General de Diversidad Biológica - Museo de Historia Natural, UNMSM.

Pacheco V. 2002. Mamíferos del Perú. In: G. Ceballos y J. Simonetti, eds. Diversidad y conservación de los mamíferos neotropicales. Conabio-UNAM. México, D.F. Pp. 503-550.

Pacheco V., R. Cadenillas, E. Salas, C. Tello \& H. Zeballos. 2009. Diversidad y endemismo de los mamíferos del Perú. Revista Peruana de Biología 16(1):5-32.

Regan H.M., L.A. Hierl, J. Franklin, D.H. Deutschman, H.L. Schmalbach, C.S. Winchell \& B.S. Johnson. 2008. Species prioritization for monitoring and management in regional multiple species conservation plans. Diversity and Distributions 14(3):462-471.

Soto K. 1999. Efectos del Niño 1997-98 sobre el ciclo reproductivo del lobo marino chusco Otaria byronia en las Islas Ballestas, Pisco. Perú. Tesis para optar el título de biólogo. Universidad Nacional Agraria La Molina.

Soto K.H., A.V. Trites \& M. Arias-Schreiber. 2004. The effects of prey availability on pup mortality and the timing of birth of South American sea lions (Otaria flavescens) in Peru. Journal of Zoology 264(4):419-428.

Tisdell C. \& H. Swarna Nantha. 2007. Comparison of funding and demand for the conservation of the charismatic koala with those for the critically endangered wombat Lasiorhinus krefftii. Biodivers Conserv 16:1261-1281.

Wilson D.E. \& R.A. Mittermeier (eds.) 2009. Handbook of the Mammals of the World. Carnivores. Vol. I. Lynx Editions, Barcelona.

Wilson E.O. 2000. On the future of conservation biology. Conservation Biology, 14:1-3. 